\title{
Projecting into the Third Dimension: 3D Ore Mineralogy via Machine Learning of Automated Mineralogy and X-Ray Microscopy
} \author{
and Richard J. Harrison ${ }^{1}$ \\ 1. Department of Earth Sciences, University of Cambridge, Cambridge, UK. \\ 2. Department of Earth Science and Engineering, Imperial College, London, UK. \\ 3. Carl Zeiss X-ray Microscopy, Pleasanton, CA, USA. \\ 4. Earth and Ocean Sciences, National University of Ireland, Galway, Ireland. \\ * Corresponding author: mb977@cam.ac.uk
}

Matthew R. Ball ${ }^{*}$, Joshua F. Einsle ${ }^{2}$, Matthew Andrew ${ }^{3}$, David D. McNamara ${ }^{4}$, Richard J.M. Taylor ${ }^{1}$

Analytical capabilities of scanning electron microscopy (SEM) and light microscopy (LM) allow accurate phase identification in two dimensions (2D), using a diverse set of characteristics. Chemistry can be accessed directly from energy (or wavelength) dispersive X-ray spectroscopy (EDS/WDS) and automated mineralogy (AM), or indirectly from backscatter electron (BSE) intensity. Particle shape and morphology can be quantified using image analysis tools on BSE, secondary electron (SE) or reflected light micrographs. Crystallographic data is now also widely available through electron backscatter detector (EBSD) data, which can provide data on stress and strain within a sample. However, with the exception of highly destructive 'slice and view' experiments [1], these analytical techniques have so far been limited to 2D. Here we aim to combine analytical data, in this case ZEISS Mineralogic Mining, a form of AM, with three-dimensinal (3D) X-ray microscopy (XRM) data, collected using the ZEISS VERSA, to display the full 3D mineralogy and texture of an ore body.

The 3D texture and morphology of grains in economic geological samples can have important implications for the extraction of elements. The Lisheen deposit is a $\mathrm{Zn}-\mathrm{Pb}-\mathrm{Ag}$ deposit containing around $22 \mathrm{Mt}$ of ore hosted by sulphide lenses [2]. Originally mined for $\mathrm{Pb}$ and $\mathrm{Zn}$, this ore body is being re-evaluated for its potential as a source of 'energy critical elements', such as La, Ce, Co and Li. Here we apply an XRM AM method, previously applied to simple systems such as sandstones, to this system to extract mineral phases in $3 \mathrm{D}$, across a range of crystal sizes and morphologies, shown in Figure 1. A half core of the Lisheen was cut into two sections, an upper and lower section, creating two half cores with two perpendicular polished faces which roughly separate the core into coarse- and fine-grained regions.

Mineralogic Mining applied to both the top and front face of both core sections is taken to reveal the 2D phase distribution of mineral phases. XRM follows this, which yields the 3D density distribution of the grains within the sample. Using these two datasets as separate training sets, machine learning is applied to the XRM dataset, to reproduce virtual slices of mineral phase maps throughout the 3D distribution. Virtual slices based on one face are then directly compared to the real mineral phase maps of the other face. The whole workflow is shown schematically in Figure 2. This allows us to exploit the unique capability of AM to chemically separate phases of similar density, with the non-destructive 3D imaging of XRM. This has wide ranging applications for ore geology and geoscience, allowing for 3D textural studies and economic element distributions to be fully exploited [3]. 
References:

[1] I Lascu et al., Journal of Geophysical Research: Solid Earth 123 (2018), p. 7285.

[2] MW Hitzman, PB Redmond and DW Beaty, Economic Geology 97 (2002), p. 1627.

[3] The authors acknowledge ZEISS for instrument access.

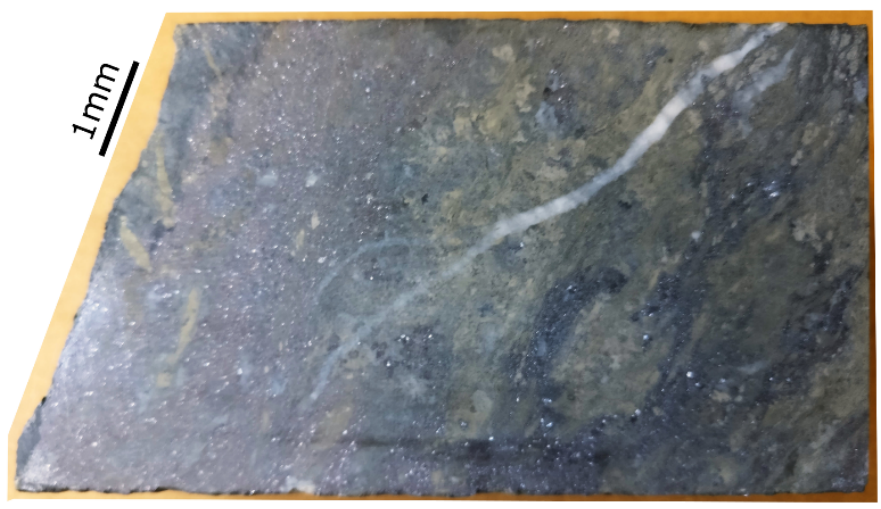

Figure 1. The upper section of the Lisheen half-core, showing the variation in grain size and mineralogy as well as the defined shear texture across a vein.

\section{Sample}

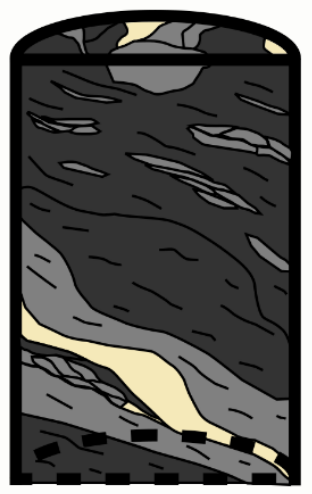

Automated Mineralogy

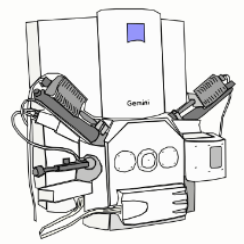

SEM-EDS


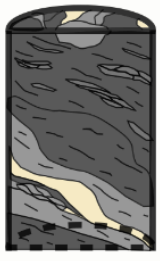

XRM
Reconstructed 3D Mineralogy

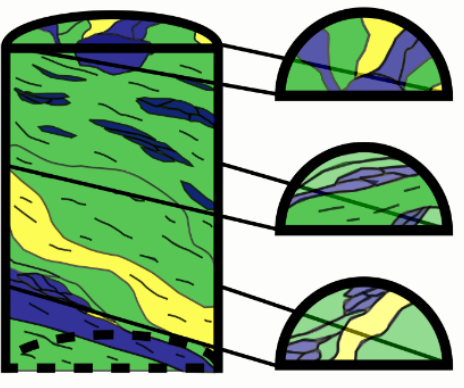

Figure 2. Schematic workflow of the $2 \mathrm{D}$ to $3 \mathrm{D}$ analytical process. The machine learning uses an iterative process to converge on the true 3D mineralogy using virtual slices which can be compared to real AM maps which were not used for the reconstruction as an internal self-consistency check. 